\title{
Bioinformatics in Drug Discovery a Review
}

\author{
N. Siddharthan, M. Raja Prabu and B. Sivasankari
}

\begin{abstract}
The drug discovery process was beginning in $19^{\text {th }}$ century by John Langley in 1905 when he proposed the theory of respective substances. Drug discovery is the step-bystep process by which new candidate drugs are discovered. Bioinformatics deals with the exponential growth and the development in primary and secondary databases like nucleic acid sequences, protein sequences and structures. Drugs are usually only developed when the particular drug target for those drugs' actions have been identified and studied. Drug target validation helps us to check the potential for failure in the clinical testing and approval phases. Drug discovery also involves several phases from target identification to preclinical development. The aim of this study is to produce lead compounds i.e., new analogs with improved potency. The study of the promising compound can be divided into two different stages - preclinical pharmacology and clinical pharmacology.
\end{abstract}

There are several key areas in bioinformatics regarding $C A D D$ research.

Keywords--- Drug Discovery, Target Identification, Target Validation, Clinical Pharmacology, CADD

\section{INTRODUCTION}

$\mathrm{T}$ HE drug discovery process was beginning in $19^{\text {th }}$ century by John Langley in 1905 when he proposed the theory of respective substances. The first rational development of synthetic drugs was carried out by Paul Ehrlich (father of modern chemotherapy) and SacachiroHata who produced arsphenamine(Salvarsan) in 1910 by structure activity relationship from atoxyl used in sleeping sickness (trypanosomiasis) and syphilis [1]. Drug discovery is the stepby-step process by which new candidate drugs are discovered. Normally, pharmaceutical companies follow well-established pharmacology and chemistry-based drugdiscovery approaches, and face various difficulties in finding new drugs [2].The aim of drug discovery is to produce more and more drugs in a short period of time with low risk in bioinformatics [3]. In fact, now there is an existence of new, separate field, known as computer-aided drug design (CADD), [4],[5].

N. Siddharthan, Department of Biology, Gandhigram Rural InstituteDeemed University, Gandhigram-624 302, Dindigul (District), Tamilnadu. Email:siddhanabi11@gmail.com

M. Raja Prabu, Department of Biology, Gandhigram Rural InstituteDeemed University, Gandhigram-624 302, Dindigul (District), Tamilnadu.

B. Sivasankari, Department of Biology, Gandhigram Rural InstituteDeemed University, Gandhigram-624 302, Dindigul (District), Tamilnadu. DOI: 10.9756/IJRAS.8099

\section{BIOINFORMATICS}

Bioinformatics deals with the exponential growth in biological data have led to the development of primary and secondary databases of nucleic acid sequences, protein sequences, and structures. Some of the well-known databases include GenBank, SWISS-PROT, PDB, PIR, SCOP, CATH, etc., these databases are available as public domain information and hosted on various Internet servers across the world. The basic research is done by using different databases along with the help of sequence analysis tools such as BLAST, FASTA, CLUSTALW, etc. The modeled structures are viewed using WebLab, MOLMOL, Rasmol. Bioinformatics is considered as significant in understanding the complex mechanisms of the cell. Moreover Bioinformatics is very useful for biomedical investigators to test the clinical samples. The "Clinical Informatics" is the complete process of data collection to analysis the samples and the results [6].

\section{DRUG DISCOVERY}

The drug discovery process can be described as the identification and validation of a disease target and the discovery and development of a chemical compound to interact with that target [7].Pharmaceutical industry need multidisciplinary informatics systems such as High Throughput Screening(HTS) data, Computational Chemistry, Combinatorial Chemistry, ADME Informatics, Cheminformatics, Toxicology, Metabolic Modeling, Bioinformatics in medical substance, Drug Discovery and Metabolism and so on. Information way in and news between different departments like the development and discovery [8].

\section{DRUG TARGET IDENTIFICATION}

The current approach is to identify the biologically active candidates [9]. Drugs are usually only developed when the particular drug target for those drugs' actions have been identified and studied. The number of potential targets for drug discovery process is increasing exponentially. Mining and warehousing of the human genome sequence using bioinformatics has helped to define and classify the nucleotide compositions of those genes, which are responsible for the coding of target proteins, in addition to identifying new targets that offer more potential for new drugs [10], [11]. This is an area where the human genome information is expected to play a master role [12]. Drug developers developed the unnatural good quality of more genes are identified and the drug discovery cycle becomes more data-intensive [13]. Bioinformatics is used to identify and analyse more and more biological drug targets; thus expected to greatly increase the breath of possible drugs in the pipelines of pharmaceutical companies [9], [3]. 


\section{DRUG TARGeT VALIDATION}

Bioinformatics also provides strategies and algorithm to predict new drug targets and to store and control available drug target information. After the discovery of "potential", drug targets, there is an insignificant need to establish a strong association between a putative target and disease of interest [12]. The establishment of such a key association provides justification for the drug improvement process this process is known as target validation. It is an area where bioinformatics play a vital role (Fig. 1). The possibility for failure in the clinical testing and approval phases can be moderated by Drug target validation [9], [14], [15].

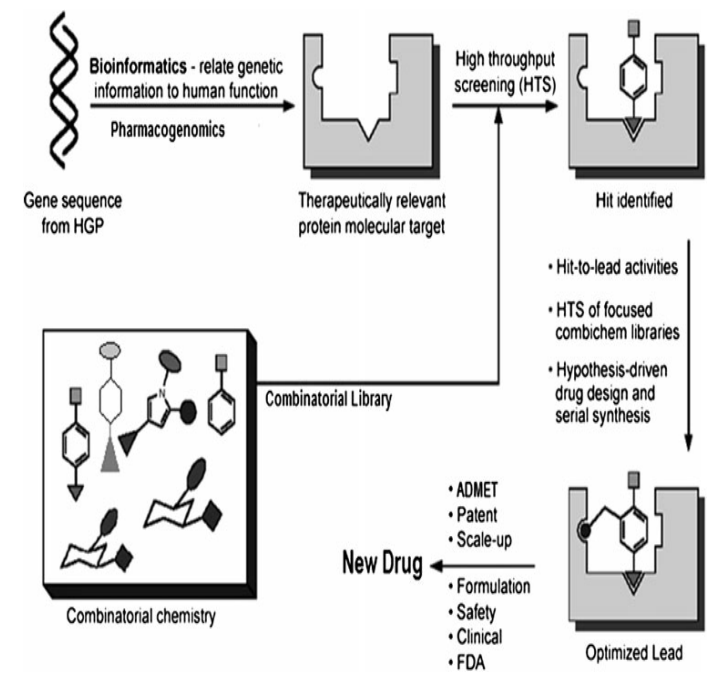

Fig. 1: Role of Bioinformatics in Various Stages of Drug Discovery Process

\section{HIT TO LEAD SELECTION}

Drug discovery involves different phases from target identification to preclinical development. In modern drug discovery involves the process of transforming small molecules of protein function into high content lead activities [16]. Most commonly hit compounds are derived by High Throughput Screening (HTS). HTS a high-tech approach for drug discovery in current trend to show how selective the compounds are for the chosen target and it is more and more gaining popularity among industrial Researchers[17]. This program have the potential to screen 10,000 compounds per day, while Ultra High Throughput Screening (UHTS) can screen upto 100,000 assays per day [18].

Recently, techniques like Particle Count Measurements for varying sample concentration, 2D fluorescence intensity distribution analysis for fluorescence interference or color quenching corrections in scintillation proximity assays (SPAs) have been proposed to eliminate some artifacts[19].

\section{Lead Optimization}

It follows the lead finding process. The lead aim to synthesize lead compounds, new analogs with improved potency. Reduce of target activities, and to optimize lead with other properties such as Selectivity, metabolic stability, etc. This optimization is proficient through chemical modification of the hit structure, with modifications chosen by employing structure-activity analysis (SAR) as well as structure based design if structural information about the target is available [8].The lead compound should have a good drug likeness \& would not interfere with the cytochrome P450 enzymes or with the P-glycoprotein.

\section{Drug Development}

Once a new chemical was identity and discovered it has to be subjected to the development process. The ability of chemical is mostly carried out in the R \& D divisions of the pharma companies. The structure activity relationships (SAR) are determined. The compounds are divided into two stages preclinical pharmacology (animal studies) and clinical pharmacology (human studies)[20].

\section{Preclinical Studies}

The candidate drug is subjected to extensive pharmacological testing invitro and invivo on animal models (mice, rats, pigs, dogs). Major areas of research are[21]:Acute, subacute and chronic toxicity studies (toxicity profile) The rapeutic index (safety and efficacy evolution): it is the ratio of median effective dose(ED50).

Absorption, distribution, metabolism \&elimination ADME studies (pharmacokinetics)

\section{Clinical Studies}

Clinical studies determine the metabolic and pharmacologic activity of the drug in humans, side effects due to increasing the normal dose to higher doses, to gain early evidence on efficacy. About $90 \%$ of drug candidate entering into clinical trials were failed. In 1991, the main reason for failure was problems in PK/bioavailability (40\%) followed by lack of efficacy $(30 \%)$ and toxicology(12\%). In 2000, the main reason for failure was lack of efficacy (27\%), followed by commercial and market reasons (21\%)[22]. There are four phases of clinical trials.

Phase 1: Clnical Phramacology Evolution

Phase 2: Controlled Clinical Evolution

Phase 3: Extended Clinical Evolution

Phase 4: Surveillance During Post Marketing General Clinical Use

\section{Recent Techniques}

Recent techniques in bioinformatics in drug discovery includes Computer-aided drug design (CADD). Computer aided drug design is a method to simulate drug receptor interactions. CADD methods are dependent on bioinformatics tools, applications and databases. There are several key areas in bioinformatics regarding CADD research [20]. Bioinformatics techniques are used in two different phases of drug discovery to extract interesting information and find important genes and proteins speeding the process of drug discovery, this method gives the accuracy of analysis and its cost is low. Gene identification is a very fundamental and important technique among them [10]. 


\section{CONCLUSION}

Since 1990s, the drug discovery process has been revolutionized with the introduction of some newer techniques in molecular biology, biotechnology, genomics and bioinformatics. Due to the speed, new trends and faster development in drug discovery leads to greater success in the bioinformatics tools. HTS is considering as the powerful technique which is used to speed up the complete screening process. Target oriented development is a plus point and receptors especially G-protein coupled receptors (GPCRs) has been successfully targeted. Despite all this, there has been a steady decline in the number of new drugs and still drug discovery a lengthy, expensive, difficult and inefficient process with low rate of new therapeutic discovery.

\section{ACKNOWLEDGEMENT}

The author wishes to thanks for the Department of Biology in The Gandhigram rural university-Deemed university, Dindigul and also thanks for our beloved friends for their full support.

\section{REFERENCE}

[1] Gereth Thomas, "Medicalchemistryan introduction", $2^{\text {nd }}$ edition John Wiley Publication, Pp.5-72, 2007.

[2] M.Iskar, G. Zeller, Zhao XM, V.Van Noort, P. Bork, "Drug discovery in the age of systems biology: the rise of computational approaches for data integration”, Curr Opin Biotechnol 23, Pp.609-616, 2012.

[3] S.S. Ortega, L.C. Cara, M.K. Salvador, "In silico pharmacology for a multidisciplinary drug discovery process", Drug Metabol Drug Interact 27, Pp.199-207, 2012.

[4] C.M. Song, S.J. Lim, J.C. Tong, "Recent advances in computer aided drug design", Brief Bioinform, 10, Pp.579-591, 2009.

[5] A. Speck-Planche, M.N. Cordeiro, "Computer-aided drug design, synthesis and evaluation of new anti-cancer drugs", Curr Top Med Chem. [Epub ahead of print], 2013.

[6] D.E. Clark, S.D. Pickett, "Computational methods for the prediction of 'drug-likeness", Drug Discovery Today.

[7] A. Emst, D. Obrecht, "Case studies of parallel synthesis in hit identification, hit exploration, hit-to-lead and lead-optimization programs, in High-Throughput Lead Optimization in Drug Discovery", USA-CRS Press, Pp.99-116, 2008.

[8] "Drug discovery hit to lead", Available from in Wikipedia.org/wiki/ Drug_discovery_hit_to_lead, cited march 262012.

[9] P.Whittaker, "What is the relevance of bioinformatics, to pharmacology?", Trend PharmacolSci, 24, Pp.434-439, 2003.

[10] Y. P. Chen, F. Chen, "Identifying targets for drug discovery using bioinformatics", Expert OpinTherTarg, 12, Pp.383-389, 2008.

[11] P. Katara, A.Grover, H. Kuntal, V.Sharma, "In silico prediction of drug targets in vibrio cholera", Protoplasma 248, Pp.799-804, 2011.

[12] Y. Yamanishi, M. Kotera, M. Kanehisa, S. Goto, "Drug-target interaction prediction from chemical, genomic and pharmacological data in an integrated framework", Bioinformatics 26:i246-i254, 2010.

[13] M. Loh, R. Soong, "Challenges and pitfalls in the introduction of pharmacogenetics for cancer", Ann Acad Med Sin gap 40, Pp.369-374, 2011.

[14] E. Ratti, D. Trist. "Continuing evolution of the drug discovery process", Pure Appl. Chem. 73, Pp.67-75, 2001.

[15] J. Gilbert, P. Henske, A. Singh, "Rebuilding Big Pharma's Business Model", In vivo Business and Medicine Report 21, Pp 10, 2003.

[16] K.H. Bleicher, H.J. Bohm, K. Muller, A.I. Alanine, "Hit and lead generation: beyond High-throughput Screening", Net Rev Drug Discov, 2(5), Pp.369-78, 2003.

[17] E.A.Martis, R.Radhakrishnan, R.R. Budve, "High Throughput Screening. The Hits and Leads of Drug Discovery", An overview Journal of Applied Pharmaceutical Science: 1(1), Pp.02-10, 2011.

[18] Heyses, "Quantifying bioactivity on a large scale quality assurance and analysis of multiparametric ultra-HTS dates, JALA, 10, Pp.207-212, 2005.
[19] Y.W.Park, R.T.Cummings, Wu L, S. Zheng, P. M. Cameron, A. Woods, D.M.Zaller, "Homogeneous proximity tyrosinekinase assays; Scintillation proximity assay versus homogeneous time resolved fluorescence", Anal. Biochem, 269, Pp:94-104, 1999.

[20] A.Mohd, D.A. Jyoti. Current Trends in Drug Discovery: Target Identification to Clinical Development of the Drug, IRJP, 3(4). ISSN 2230-8407, 2012.

[21] X.Chen, E. Jorgenson and Cheung, "St. New tools for functional genomics analysis", Drug Discov Today; 14, Pp:754-760, 2009.

[22] Barar, "Essentials of Pharmacotherapeutics", $3^{\text {rd }}$ edition S.Chandand company Publication New Delhi, Pp.108-120, 2004. 